\title{
Applying Information Technology-Based Knowledge Management (KM) Simulation in the Airline Industry
}

\author{
Abdullah M. Al-Ansi \\ Research Scholar, Thamar University, Yemen \\ ebrar.ansi@yahoo.com
}

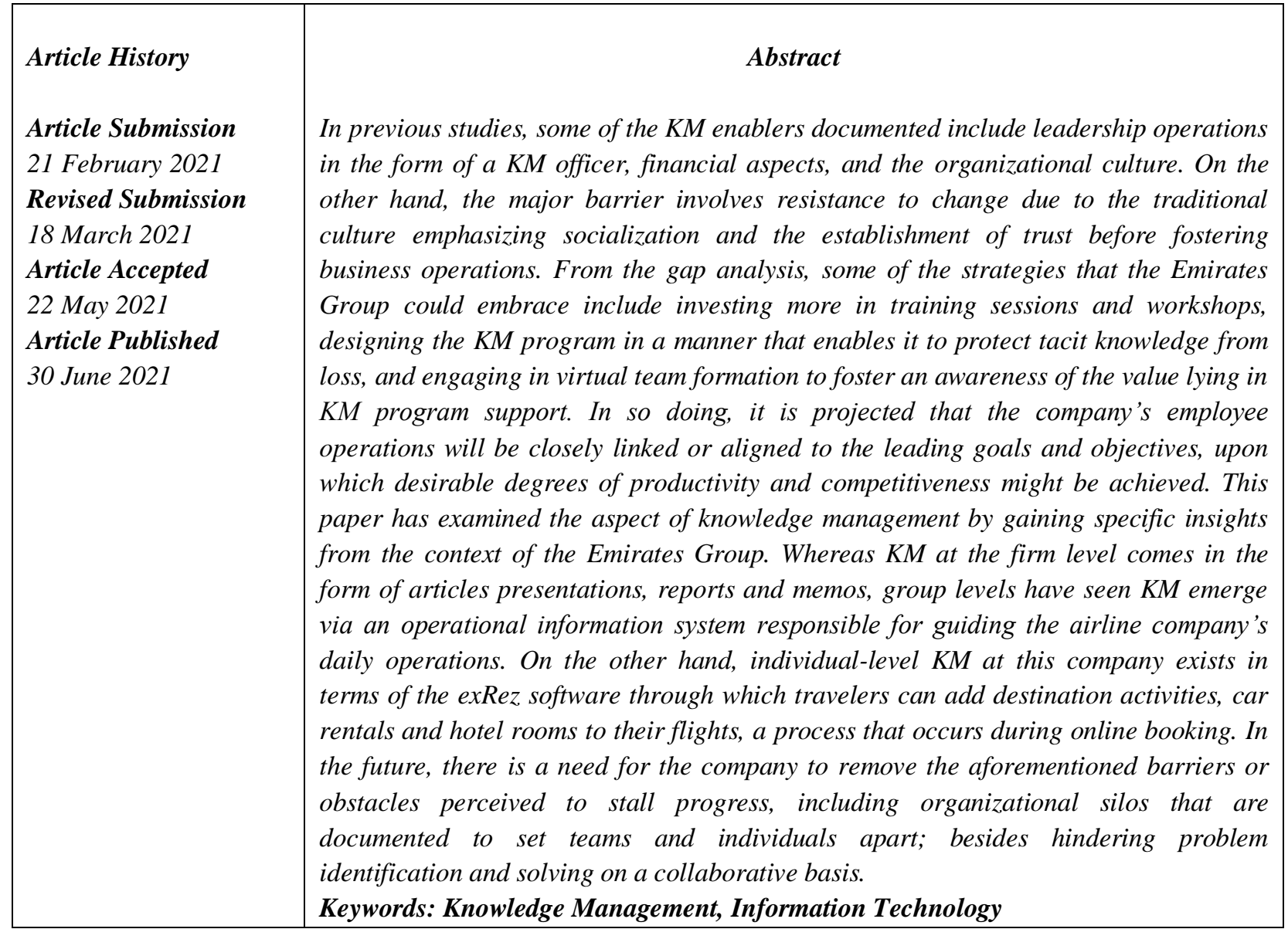

\section{Introduction}

In the context of the United Arabs Emi rates (UAE), it has been observed that the business arena is experiencing dramatic shifts from a state of labour-based operations to knowledge-based operations. According to Yozgat and Rofcanin (2015), sustainable and long-term competitive advantage in this context lies in skills as the leading resources towards surviving stiff competitive in the local, regional, and global marketplaces. Operating as an aviation holding firm, the Emirates Group is based in Dubai and headquartered in Garhoud. The company operates fleets that exceed 250 wide-bodied aircrafts and the latter fly to more than 140 destinations in six continents. The company has also employed more than 62,000 workforces in all its associated firms and business units, becoming a renowned employer in the region of the Middle East (the Emirates Group 2017). In relation to knowledge management, the Emirates Group (2017) observed that the process entails the practice of handling knowledge assets towards value creation to meet the strategic and tactical requirements of a firm. Therefore, it can be inferred that knowledge management seeks to foster the flow of knowledge and information between and to the right people in a company. The motivation is to deliver information at the right cost, in the right format, and at the right time for effective and efficient acting towards the organisation's value creation. At the Emirates Group, the leading 
knowledge management initiative lies in the practice of a movement level constituting the decision-support system and the management information system. According to the Emirates Group (2017), the management information system at the Emirates Group focuses on internal information sources in which data is taken from transaction processing systems before being summarized into management reports that assume a series. Indeed, management information systems as knowledge management practices at the Emirates Group occur at the organization level and involve data regarding articles, presentations, reports, and memos that are stored in repositories where they can be retrieved with ease (the Emirates Group 2017). At the group level, the company utilizes an operational information system to guide daily operations of this airline firm. Specific data entails crew, passengers, and lights. Lastly, knowledge management at the individual level assumes the form of the ezRez software that enables travelers to add destination activities, car rentals and hotel rooms to their flights during online booking (the Emirates Group 2017).

\section{Methodology}

This model of KM advocates for the existence of two types of knowledge. On the one hand, tacit knowledge is acquired via the people's experience and communicated indirectly through analogy and metaphor. On the other hand, explicit knowledge is perceived as that which is contained in procedures and manuals (Nonaka \& Takeuchi 1995). During knowledge conversion, the model holds that socialization entails sharing tacit knowledge via participation in informal and formal communities, practice, imitation, and observation. At the Emirates Group, knowledge management at the individual level could be steered by ensuring that senior leaders walk the talk and act as role models before allowing other employees to act in the required manner, having observed and imitated these senior leaders. The model proceeds to indicate that externalization entails tacit knowledge articulation into concepts that are explicit (Sabherwal \& Becerra-Fernandez 2003). At the Emirates Group, this step could be achieved by holding seminars and conferences to foster knowledge creation and sharing via feedback provision. The third step in the selected model involves combination whereby concepts are integrated into knowledge systems. Lastly, internalization implies that explicit knowledge is embodied in to tacit knowledge. At the Emirates Group, knowledge management at the organization level could assume what is advocated in this model by fostering brief executive summaries, conducting trend analyses, and producing review reports. The selected KM model is summarized in the figure below.

\section{Results and Discussion}

Various reasons or perceived benefits account for the endless effort to foster knowledge management at the Emirates Group. For instance, the motivation to promote $\mathrm{KM}$ has been found to lie in the attribute of improved service delivery, productivity, and efficiency. According to Chen and Huang (2009), knowledge management at the Emirates Group is also motivated by the perceived benefits of facilitating effective communication of knowledge among task forces, an avoidance of overlap while performing certain tasks, the capacity to assure sound policy making and improved internal decision-making, and increased transparency. Various KM enablers exist at the company level. These enablers range from leadership to financial aspects and organizational culture (Dalkir 2011). The following figure highlights the factors influencing the degree of success in knowledge management at the Emirates Group.

From the leadership perspective, the presence of a KM officer at the Emirates group is seen to play a leading role in facilitating the practice. On the other hand, the financial resources as KM enablers have ensured that sufficient allocation is achieved to allow for the establishment of institutional, technological and human capabilities required towards the successful operation of the KM program (Dobre 2012). Hence, a sufficient annual budget targeting the KM program is seen to promote the initiative and has ended up creating necessary conditions through which employees are prompted to share knowledge. Technological instruments in terms of sophisticated IT systems form another KM enabler due to their capacity to store knowledge that has been captured. For instance, the company relies in data mining and data warehouse tools to foster knowledge storage and sharing. Online collaboration tools have also played an additionally beneficial role. Human resources via the presence of an adequate employee group 
form an additional KM enabler at the Emirates Group (Holsapple \& Wu 2011). Indeed, the central objective lies in the exploitation of a varied talent pool towards improved quality in the wake of a diverse knowledge base.

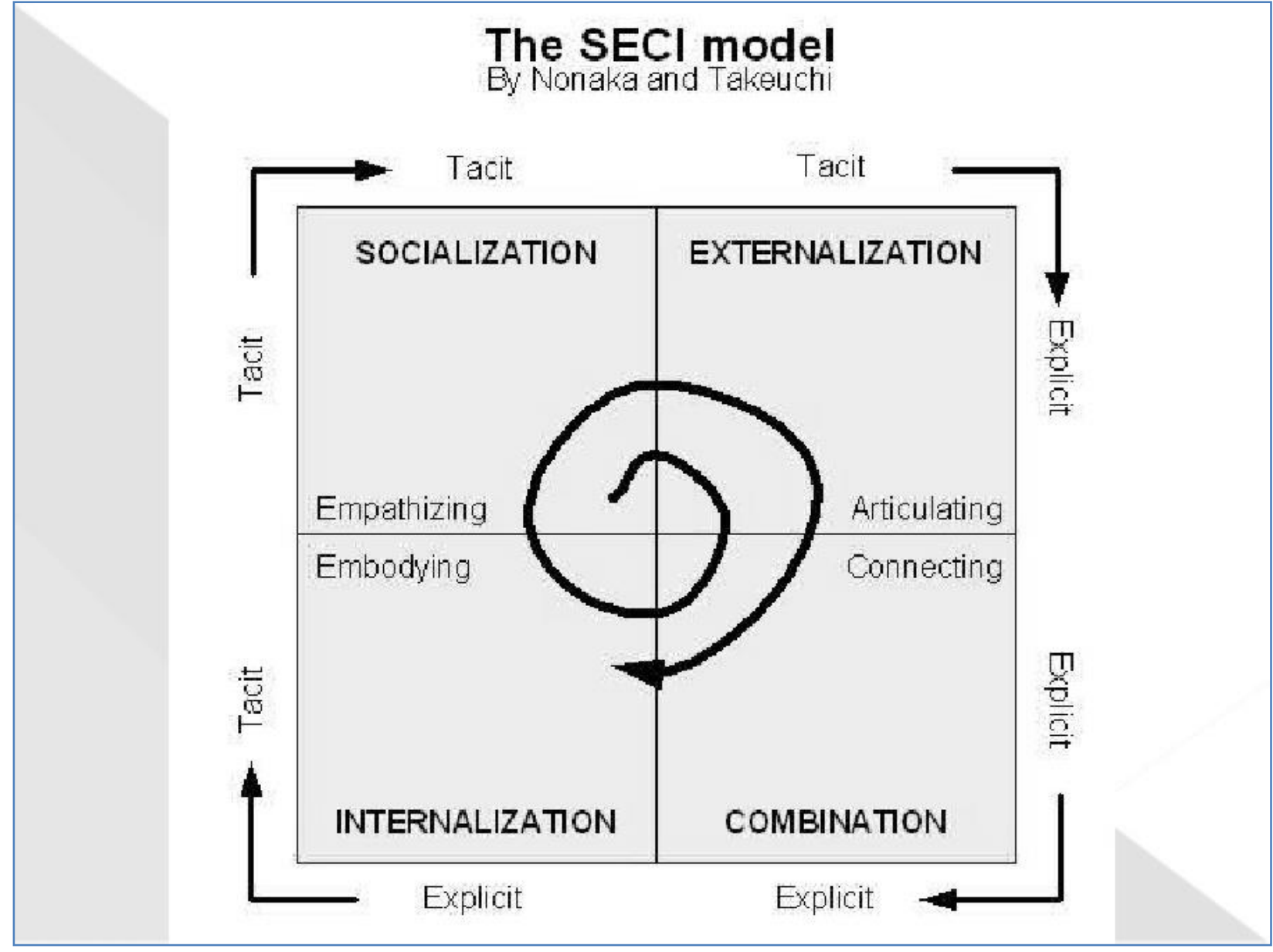

Figure number is missing

Despite the aforementioned promising trends, knowledge management at the Emirates Group has not gone without challenges. For example, knowledge transfer and sharing is seen to be hindered by the dominant organizational culture characterizing the Arab world. The eventuality has been a state of severe resistance among sections of workforces. According to Vaccaro, Parente and Veloso (2010), Arabs are among populations perceived to be the most resisting when it comes to knowledge sharing. The resistance is attributed to issues such as the traditional culture emphasizing socialization and trust establishment prior to engaging in business activities (including KM), the difficulty and complexity of knowledge sharing in the Arab environment, and hindrances emerging from cultural and social beliefs (Yang, 2010). The overall outcome has been a situation in which the lacking interpersonal trust between the management and employees has stalled progress in the Emirates Group's KM program.

At the Emirates Group, the leading dilemma is the state of lack of clarity and vagueness of the KM concept to the majority of service users. This trend is attributed to the fact that the concept has been introduced recently and is yet to gain an in-depth adoption and implementation in the selected context, as well as wide-scale acceptance among community members served by the airline company (Yozgat \& Rofcanin 2015). Therefore, the fact that KM has been introduced recently implies that the program calls for time not only to become clear to the service user groups but also to mature. These mixed outcomes imply that there is a need for the company to invest more in the organization of training sessions and workshops that involve task forces. In so doing, an understanding of what KM stands for might be achieved and its resultant and beneficial or crucial role emphasized towards firm sustainment 
and development. The recognition of the crucial role played by tacit knowledge is also seen to be lagging behind the expected level and the eventuality has been a failure to acknowledge the critical role it plays when capture, documented, and shared at the Emirates Group (Chen \& Huang 2009). To address this gap, this paper recommends that the company the design of the KM program seeks to protect tacit knowledge from loss, ascertaining its existence at the firm even in situations where sections of workforces decide to leave or are transferred. In so doing, the expected outcomes of the KM program will be achieved.

Whereas it was acknowledged earlier that the company's leadership plays the critical role of a KM enabler, most of the regional workforces are yet to gain an adequate awareness of the important role it plays in the creation of the right conditions responsible for selecting, organizing, conserving, disseminating and transferring knowledge towards strategic objective achievement. This lack of awareness has ended up compromising the Emirates Group's effort geared towards KM adoption and success as a means for realizing sustainable progress (Dalkir 2011). From the findings, it is recommended that the practices and values associated with the KM program are promoted through virtual team formation in which members could share knowledge in a structure that is well established, coherent and systematic. Lastly, the Emirates Group is seen to have pursued technological instruments that are advanced (such as data mining techniques) for purposes of storing explicit knowledge that has been created or captured but the instruments are yet to unpack tacit knowledge that remains embedded in individuals (Dobre 2012). To address this deviation, there is a need for the company to remove the aforementioned barriers or obstacles perceived to stall progress, including organizational silos that are documented to set teams and individuals apart; besides hindering problem identification and solving on a collaborative basis.

\section{Conclusion}

In summary, this paper has examined the aspect of knowledge management by gaining specific insights from the context of the Emirates Group. Whereas KM at the firm level comes in the form of articles presentations, reports and memos, group levels have seen KM emerge via an operational information system responsible for guiding the airline company's daily operations. On the other hand, individual-level KM at this company exists in terms of the exRez software through which travelers can add destination activities, car rentals and hotel rooms to their flights, a process that occurs during online booking among customer bases.

\section{References}

[1] Chen, C. \& Huang, J. (2009). Strategic human resource practices and innovation performance - the mediating role of knowledge management capacity. Journal of Business Research, 62(1), 104-114

[2] Dalkir, K. (2011). Knowledge management in theory and practice (2nd Edition). Cambridge, MA: MIT Press.

[3] Dobre, O. (2012). Managing human resources in the knowledge-based economy. Review Of Applied SocioEconomic Research, 3(1), 68-76

[4] Holsapple, C. \& Wu, J. (2011). An elusive antecedent of superior firm performance: The knowledge management factor. Decision Support Systems, 52(1), 271-283

[5] Nonaka, I. \& Takeuchi, H. (1995). The Knowledge-Creating Company. New York: Oxford University Press

[6] Sabherwal, R. \& Becerra-Fernandez, I. (2003). An Empirical Study of the Effect of Knowledge Management Processes at Individual, Group, and Organizational Levels. Decision Sciences, 34 (2): 225260

[7] The Emirates Group. (2017). The Emirates Group Annual Report 2016-2017. Retrieved on June 23, 2017 from https://cdn.ek.aero/downloads/ek/pdfs/report/annual_report_2017.pdf

[8] Vaccaro, A., Parente, R. \& Veloso, F. M. (2010). Knowledge management tools, inter-organizational relationships, innovation and firm performance. Technological Forecasting \& Social Change, 77(7), 10761089 
[9] Yang, J. (2010). The knowledge management strategy and its effect on firm performance: A contingency analysis. International Journal of Production Economics, 125(2), 215-223

[10] Yozgat, A. F. \& Rofcanin, T. (2015). Examining organizational innovation and knowledge management capacity: The central role of strategic human resources practices (SHRPs). Procedia - Social and Behavioral Sciences, 181, 377-387 